\title{
MONITOREO DE LA FOTODEGRADACIÓN BAJO IRRADIACIÓN ULTRAVIOLETA DE $\alpha$-TOCOFEROL MEDIANTE ESPECTROSCOPÍA DE ABSORCIÓN ÓPTICA
}

\author{
MONITORING OF THE PHOTODEGRADATION UNDER ULTRAVIOLET IRRADIATION OF $\alpha$ - \\ TOCOPHEROL THROUGH OPTIC ABSORPTION SPECTROSCOPY
}

${ }^{1}$ José Antonio Tiburcio Moreno, ${ }^{2}$ Lorenzo Echevarria, ${ }^{3}$ Carlos Diaz, ${ }^{4}$ Florencio Hernandez, ${ }^{5}$ Juan José Alvarado-Gil

\begin{abstract}
RESUMEN
En este trabajo se presenta un estudio sobre el proceso de fotodegradación de $\alpha$-Tocoferol bajo irradiación ultravioleta, para dos diferentes longitudes de onda. Los resultados muestran que los espectros de absorbancia de la molécula en estudio. En su forma, químicamente pura, muestran un decrecimiento en sus picos de absorción y siguen una cinética de primer orden, la cual difiere de su versión comercial (dl-a-tocoferol). En esta forma, esta investigación es de importancia para las aplicaciones y el monitoreo de la presencia de $\alpha$-tocoferol durante los procesos de almacenamiento y encapsulación.
\end{abstract}

Palabras clave: Absorción, a-tocoferol, irradiación ultravioleta.

\begin{abstract}
In this work we present a study on the photodegradation process of $\alpha$-Tocopherol under ultraviolet irradiation, for two different wavelengths. The results show that the absorbance spectra of the molecule under study. In their chemically pure form, they show a decrease in their absorption peaks and follow a first order kinetics, which differs from its commercial version (dl- $\alpha$-tocopherol). In this way, this research is of great importance for the applications and the monitoring of the presence of $\alpha$-tocopherol during the storage and encapsulation processes.
\end{abstract}

Keywords: Absorption, a-tocopherol, ultraviolet irradiation.

\section{INTRODUCCIÓN}

La vitamina $\mathrm{E}$ está constituida por diferentes compuestos, incluidos los tocoferoles y tocotrienoles. Estudios de la última década dan un rol específico a $\alpha$ tocoferol en el señalamiento y la regulación de genes, esto produce significantes efectos en los procesos de inflamación, proliferación de células y apoptosis que no son compartidos por otros isómeros de la vitamina E con similares propiedades antioxidantes (LemaireEwing et al., 2010). En los seres humanos, el $\alpha$ tocoferol es obtenido, principalmente, de aceites vegetales, leche, frutas y vegetales y está compuesto de cuatro formas isoméricas siendo la principal el alfa- tocoferol con relevancia importante en el sistema circulatorio humano (Feng et al., 2013).

Además, los niveles de $\alpha$-tocoferol es un indicador significante de la calidad de alimentos grasos y formulaciones relacionadas (Satue et al, 1995). Por ejemplo, en el procesamiento de algunos alimentos y condiciones de almacenamiento, reacciona con radicales peroxilos formando tocoferoxilo, tocoferil hidroquinonona y tocoferil quinone. Estos productos de degradación de $\alpha$-tocoferol exhiben poco o casi nada presencia de actividad de vitamina E (Rock et al., 1996).

\footnotetext{
${ }^{1}$ Facultad de Ciencias, Departamento Académico de Física, Universidad Nacional Jorge Basadre Grohmann, Av. Miraflores s/n CU, Tacna, Perú. E-mail: jtiburciom@unjbg.edu.pe

${ }^{2}$ Departamento de Química, Universidad Simón Bolívar, Caracas 1020A, Venezuela

${ }^{3}$ Department of Chemistry, University of Central Florida, P.O. Box 162366, Orlando, FL. 32816-2366, USA. E-mail: ediaz@ucf.edu

${ }^{4}$ The College of Optics and Photonics, CREOL, University of Central Florida, P.O. Box162366, Orlando, FL. 32816-2366, USA. E-mail: florenzi@ucf.edu

${ }^{5}$ Departamento de Física Aplicada, Centro de Investigación y de Estudios Avanzados del IPN-Unidad Mérida, Antigua carretera a Progreso km 6, 97310, YUC, México. E-mail: jjag09@yahoo.com
} 
Asimismo, la importancia de $\alpha$-tocoferol ha sido reconocida por su alta selectividad por el hígado para la transferencia de proteínas, lo cual está relacionado con la asociación con lipoproteínas y la secreción de la vitamina $\mathrm{E}$ dentro del flujo sanguíneo (Traber y Atkinson, 2007) (Morley et al., 2008). La función antioxidante de $\alpha$-tocoferol es bien conocida y ha sido descrita en varias investigaciones (Cora, Dillard, 1980) (Burton et al.,1985).

Los métodos actuales para la determinación de $\alpha$ tocoferol involucra cromatografía de líquidos (Kopec et al., 2013), lo cual es laborioso y requiere mucho tiempo y solventes para la extracción. Otros métodos incluyen procedimientos espectrométricos y fluorométricos directos (Tiburcio et al., 2012), los cuales son rápidos y han probado su eficiencia en la determinación de $\alpha$-tocoferol en bajas concentraciones. Algunas vitaminas son conocidas a ser degradadas cuando se exponen a la luz en particular el retinol (Allwood y Martin, 2000), tocoferol(Drott et al., 1991), vitamina $\mathrm{K}$ (Billion et al., 2015) y rivoflavina(Chen et al., 1983).

Las fotorreacciones principalmente, son el resultado de la exposición a la luz ultravioleta. En la práctica sugiere que como la luz del día, normalmente, contiene escasa irradiación UV en el rango adecuado, se tendría un mínimo efecto o influencia en la fotosensitividad de las vitaminas (Touitou y Godin, 2008). Sin embargo, el mecanismo químico responsable para el proceso de fotodegradación de diferentes vitaminas puede variar (Fuchs y Kern, 1998). Por ejemplo, el tocoferol puede ser oxidado rápidamente y esta reacción es activada por la luz ultravioleta (Kramer y Liebler, 1997).

La vitamina $\mathrm{E}$ es una molécula sensitiva a la luz y es degradada por procesos de fotoxidación y la consecuente pérdida de su actividad antioxidante (Sabliov et al., 2009) (Damiani et al., 2006). Ambas formas de la vitamina $E$, pura y esterificada, pueden ser expuestas a la luz durante proceso como, por ejemplo, la piel expuesta a la luz solar (Damiani et al., 2006) y preparación de alimentos refinados y procesados (Bramley et al., 2000).

Así, el estudio del proceso de fotodegradación de la forma comercial de la vitamina $\mathrm{E}$ en forma de acetato y su forma químicamente pura pueden contribuir a mejorar el entendimiento del rol de la exposición a la luz juega en la estabilidad de la vitamina E. El objetivo de este estudio fue monitorear este proceso para dos longitudes de onda específica UVC (254 nm) y UVA (365 nm), empleándose para este efecto espectroscopía de absorción óptica.

\section{MATERIAL Y MÉTODOS}

\section{Reactivos químicos}

$\alpha$-Tocoferol, en su forma pura, fue adquirida de Sigma-Aldrich y disuelta en hexano a diferentes concentraciones. La forma comercial de $\alpha$-Tocoferol fue adquirida en farmacias locales.

\section{Preparación de las muestras}

La molécula en estudio, $\alpha$-Tocoferol, tiene una pureza de 95,5\%. Se preparó una solución inicial disolviendo $20 \mathrm{mg}$ de $\alpha$-Tocoferol en $5 \mathrm{~mL}$ de hexano (concentración de la muestra $4 \mathrm{mg} / \mathrm{mL}$ ), desde esta solución se obtuvieron dos nuevas soluciones a las concentraciones de $1,0 \times 10^{-3} \mathrm{M}$ y $3,0 \times 10^{-4} \mathrm{M}$. Estas dos concentraciones son las adecuadas para obtener los espectros de absorción en el espectrómetro utilizado en esta investigación. Las soluciones fueron almacenadas en frascos de color ámbar para prevenir la influencia de la luz solar y almacenadas a la temperatura de $-20^{\circ} \mathrm{C}$.

\section{Irradiación de las muestras}

Las muestras fueron colocadas en una cubeta de cuarzo de $1 \mathrm{~cm}$ de camino óptico y selladas con parafilm para prevenir la evaporación. La irradiación UV fue llevada a cabo mediante periodos de $10 \mathrm{~min}$ para un acumulado máximo de 60 minutos, usando una lámpara de alta intensidad ultravioleta modelo ENF260C (Spectronic Corporation Westbury, USA) en el modo de trabajo de dos específicas longitudes de onda UV, $254 \mathrm{~nm}$ y $390 \mathrm{~nm}$.

\section{Medida de los espectros de absorbancia}

Los espectros de absorción lineal fueron tomados usando un espectrofotómetro de simple haz (Agilent 8453 Diode Array UV-Vis) desde $240 \mathrm{~nm}$ hasta 540 $\mathrm{nm}$ a través de una celda de cuarzo de $1 \mathrm{~cm}$ de camino óptico. Las contribuciones del solvente y la celda de cuarzo fueron sustraídas de todos los espectros. Se realizaron tres medidas de espectros de absorción, bajo condiciones experimentales similares de las dos concentraciones usadas en este estudio para asegurar que los espectros pueden ser repetidos y obtener los mismos resultados en cada caso. Los cálculos estadísticos fueron realizados mediante el software OriginPro versión 8,0 (marca registrada de OriginLab Inc.) y el ajuste teórico-experimental mediante un modelo de mínimos cuadrados.

\section{RESULTADOS Y DISCUSIÓN}

En la figura 1, se muestra los espectros normalizados teórico y experimental. El espectro teórico fue obtenido en la metodología del funcional de densidad dependiente del tiempo al nivel B3LYP con una base 6-31 $+\mathrm{G}^{* *}$, los detalles son presentados y discutidos en la referencia (Vivas et al., 2013). Se puede observar una correspondencia casi exacta entre ambos espectros, donde se nota una estructura vibracional resuelta con un pico característico centrado en $290 \mathrm{~nm}$, lo cual es atribuido a modo efectivo de vibración interno en esa región espectral. 


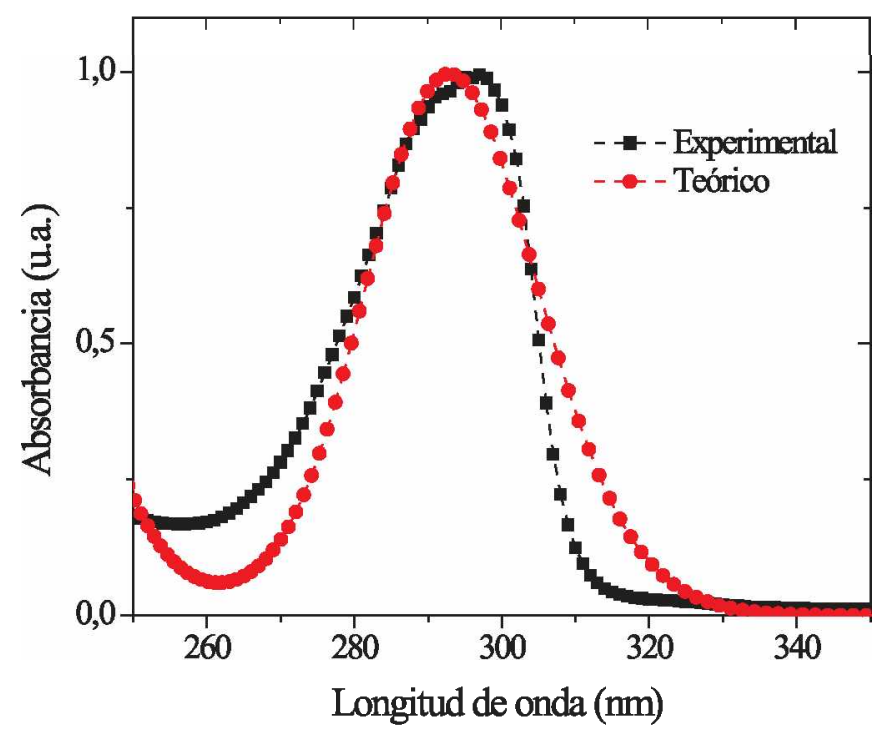

Figura 1. Espectros de absorción, teórico y experimental, de -tocoferol en una solución de Hexano a una concentración de $1.0 \times 10^{-3} \mathrm{M}$

Fuente: Elaboración propia

En la figura 2a, se presenta los espectros de absorción de $\alpha$-tocoferol a través de $60 \mathrm{~min}$ de exposición a la irradiación UV a $366 \mathrm{~nm}$. Puede ser observado que hasta los $60 \mathrm{~min}$ de tratamiento UV los espectros de absorción no presentan cambios significativos. Estos

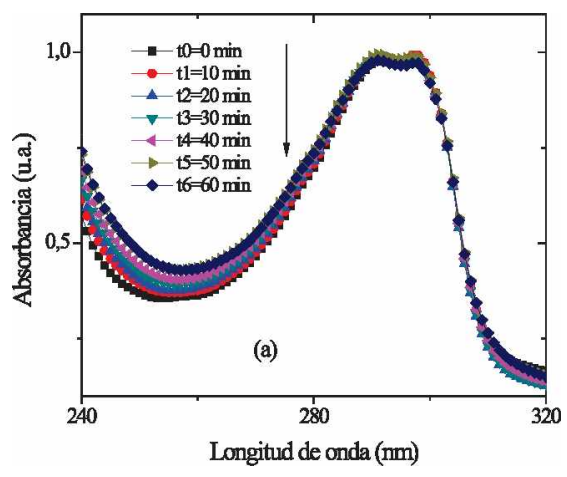

(a) resultados sugieren que $\alpha$-tocoferol no sufre foto descomposición a esta específica longitud de onda. Estos resultados concuerdan con estudios realizados anteriormente y que han sido reportados por J.A (Tiburcio et al., 2012).

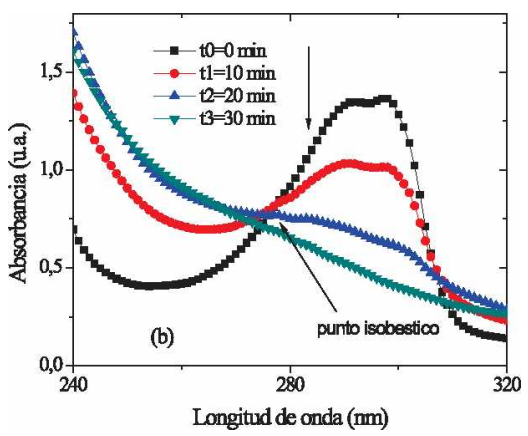

(b)

Figura 2. Efecto de la irradiación UVA (a) y UVC (b), sobre los espectros de absorbancia de -tocoferol en solución Fuente: Elaboración propia

En la figura $2 b$, se presenta los espectros de absorción de una solución similar de $\alpha$-tocoferol para una irradiación de $254 \mathrm{~nm}$ por un tiempo máximo de 30 min, se observa que los picos de absorción decrecen rápidamente hasta desaparecer completamente para el tiempo máximo de irradiación. La rápida cinética del proceso observada a esta longitud de onda corta UV es atribuida a una eficiente foto degradación. Para determinar el orden de este proceso, se graficó el logaritmo de la absorbancia máxima vs. tiempo de irradiación (figura 3). 


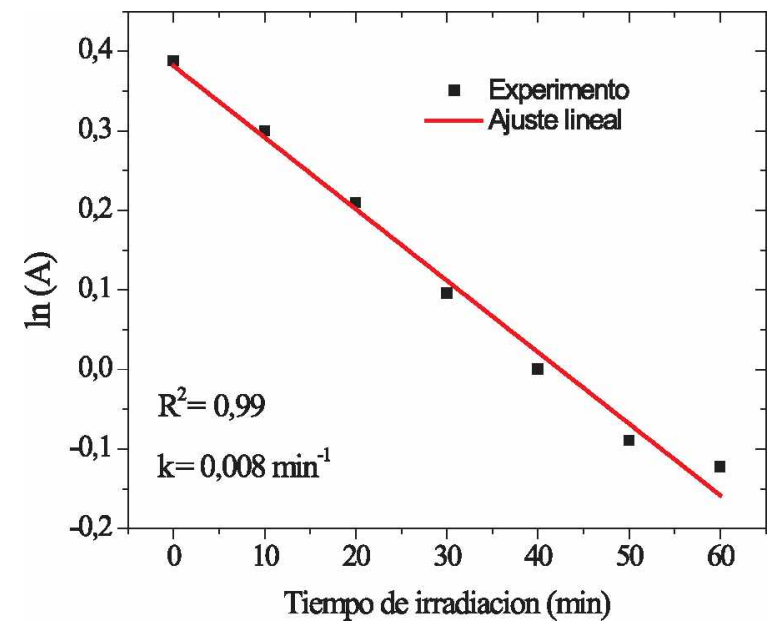

Figura 3. Absorbancia máxima vs. tiempo de irradiación y su correspondiente ajuste lineal para la cinética de foto degradación de-tocoferol

Fuente: Elaboración propia

En esta figura, se puede observar que los espectros se concentran en un punto isosbéstico, lo cual, es un indicador que la degradación de $\alpha$-tocoferol se convierte en un mismo producto, pero, a una más baja concentración, lo cual, confirma que la cinética de fotodegradación es de primer orden (Sabliov et al., 2009). En la figura 3, se graficó la relación: $\ln (A)$ vs $t$ y su correspondiente ajuste lineal, el modelo cinético corresponde a una reacción de primer orden en el reactante siguiendo la ecuación: $\ln (A)=\ln \left(A_{0}\right)-k t$. En esta ecuación, $A$ es un coeficiente que está relacionado con la concentración descrito por la ley de Beer. Los valores de las constantes asociadas con esta tasa de fotodegradacion fueron determinadas usando el ajuste lineal de la data experimental. Los valores obtenidos corresponden a las constantes son: $k=$ $\left(0,008 \pm 4,34 \times 10^{-4}\right) \mathrm{min}^{-1}$. Estos resultados fueron obtenidos estadísticamente mediante un ajuste de mínimos cuadrados tomando en consideración todos los datos obtenidos en los experimentos con un programa informático de tratamiento estadístico de datos, OriginPro 8,0 (Analysis, 2013). Los valores correspondientes a la estadística se muestran en la figura 3, y muestran claramente que $\alpha$-tocoferol es muy sensitivo la fotodegradacion por irradiación UV a corta longitud de onda.

\section{CONCLUSIONES}

El proceso de fotodegradación de $\alpha$-tocoferol, en una solución de hexano, fue monitoreado exitosamente usando espectroscopía de absorción óptica. El modelo soporta una cinética de primer orden, estos resultados confirman la unimolecularidad del proceso, el cual toma lugar a una corta longitud de onda en la región UVC. Sin embargo, se confirma que la molécula no es afectada mayormente por irradiación UVA. Los resultados reportados en este artículo representan una contribución al mejor entendimiento del proceso de foto degradación y se espera contribuir en la industria farmacéutica, de alimentos y antioxidantes naturales en sus procesos de almacenaje y encapsulación.

\section{AGRADECIMIENTO}

Nuestro agradecimiento al Laboratorio de Materiales Ópticos Nolineales y Sensores de la University of Central Florida (USA) por el apoyo brindado en el uso de equipos y reactivos usados en el desarrollo de la presente investigación.

\section{REFERENCIAS BIBLIOGRÁFICAS}

Allwood, M. y Martin, H. J. (2000). The photodegradation of vitamins $\mathrm{A}$ and $\mathrm{E}$ in parental nutrition mixtures during infusion. Clinical Nutrition, 19(5), 339-342.

Analysis, G. (2013). OriginPro 8.0.

Billion-Rey, F., Guillaumont, M., Frederich, A. y Aulagner, G. (2015). Stability of fat-soluble vitamins A (retinol palmitate), E (tocopherol acetate), and $\mathrm{K} 1$ (phylloquinone) in total parenteral nutrition at home. JPEN. Journal of Parenteral and Enteral Nutrition, 17(1), 56-60. http://doi.org/10.1177/014860719301700156

Bramley, P., Elmadfa, I., Kafatos, A., Kelly, F., Manios, Y., Roxborough, H., Wagner, K. (2000). Review Vitamin E. Journal of the Science of Food and Agriculture, 80, 913-938.

Burton. G., Doba, T., Gabe, E., Hughes, L., Lee, F., Prasad, L., K. Ingold, K. (1985). Autoxidation of biological molecules. Maximizing the antioxidant activity of phenols. Journal of the American Chemical Society, 107, 7053. 
Chen, M., Worth-Boyce, H. y Triplett, L. (1983). Stability of the vitamin B in mixed parenteral nutrition solution. Journal of Parenteral and Enteral Nutrition, 7(5), 462-464.

Cora, J., Dillard, M. y A. L. T. (1980). Respiratory Pentane: A Measure of in vivo lipid peroxidation applied to rats fed diets vrying in polysaturated fats, vitamine $\mathrm{E}$ and Selenium exposed to Nitrogen Dioxide. Toxicology Letters, 6, 251-256.

Damiani, E., Rosati, L., Castagna, R., P. C. y L. G. (2006). Changes in ultraviolet absorbance and hence in protective efficacy against lipid peroxidation of organic sunscreens after UVA irrdiation. Journal of Photochemistry and Photobiology B: Biology, 82, 204-213.

Drott, P., Meurling, S. y Meurling, L. (1991). Clinical adsorption and photodegradation of the fat-soluble vitamins A and E. Clinical Nutrition, 10, 348-351.

Feng, S., Gao, F., Chen, Z., Grant, E., Kitts, D., Wang, S. y Lu, X. (2013). Determination of alphaTocopherol in vegetable oils using a moleculary imprinted polymers-surface-enhanced Raman Spectroscopy Biosensor. Journal of Agricultural and Food Chemistry, 61, 10467.

Fuchs, J. y Kern, H. (1998). Modulation of UV-lightinduced skin inflammation by D-alpha-tocopherol and L-ascorbic acid: A clinical study using solar simulated radiation. Free Radical Biology and Medicine, 25(9), 1006-1012. http://doi.org/10.1016/S0891-5849(98)00132-4

Kopec, R., Schweiggert, R., Riedl, K., Carle, R. y Schwartz, S. (2013). Comparison of highperformance liquid chromatography for the quantitation of carotenoids, tocopherol of human plasma. Rapid Communications in Mass Spectrometry, 27, 1393-1402.

Kramer, K. y Liebler, D. (1997). UVB Induced photooxidation of Vitamin E. Chemical Research in Toxicology, 10, 219-224.

Lemaire-Ewing, S., Desrumaux, C., Néel, D. y Lagrost, L. (2010). Vitamin E transport, membrane incorporation and cell metabolism: Is alphatocopherol in lipid rafts an oar in the lifeboat? Molecular Nutrition and Food Researches, 54, 631-640.

Morley, S., Cecchini, M., Zhang, W., Virgulti, A., Noy, N., Atkinson, J. y Manor, D. (2008). Mechanisms of Ligand Transfer by the Hepatic Tocopherol Transfer Protein. The Journal of Biological Chemistry, 283(26), 17797-17804.
Rock, C., Jacob, R. y Bowen, P. (1996). Update on the biological characteristics of the antioxidant micronutrients: vitamin $\mathrm{C}$, vitamin $\mathrm{E}$, and the carotenoids. Journal of the American Dietetic Association, 96(7), 693-694. http://doi.org/10.1016/S0002-8223(96)00190-3

Sabliov, C., Fronczek, C., Astete, C., Khachaturyan, M., Khachatryan, L. y Leonardi, C. (2009). Effects of Temperature and UV Light on Degradation of alpha-Tocopherol in Free and Dissolved Form. JAOCS, Journal of the American Oil Chemists' Society, 86(9), 895-902. http://doi.org/10.1007/s11746-009-1411-6

Sabliov, C., Fronczek, C., Astete, C., Khachaturyan, M., Khachatryan, L., y L. C. (2009). No Titl. Journal of the American Oil Chemists' Society, 86, 895.

Satue, T., Huang, S. y Frankel, E. (1995). Effect of Natural Antioxidants in Virgin Olive Oil on Oxidative Stability of Refined, Bleached, and Deodorized Olive Oil. Journal of the American Oil Chemist's Society, 72, 1131-1137.

Tiburcio-Moreno, J., Marcelin-Jimenez, G., LeanosCastañeda, O., Yanez-Limon, J. y Alvarado-Gil, J. (2012). Study of the Photodegradation Process of Vitamin E Acetate by Optical Absorption, Fluorescence, and Thermal Lens Spectroscopy. International Journal of Thermophysics, 33, 2062-2068.

Touitou, E. y Godin, B. (2008). Skin nonpenetrating sunscreens for cosmetic and pharmaceutical formulations. Clinics in Dermatology, 26(4), 375-379.

http://oi.org/10.1016/j.clindermatol.2008.01.014

Traber, M. y Atkinson, J. (2007). Vitamine E, antioxidant and nothing more. Free Radical Biology and Medicine, 43, 4-15.

Vivas, M., Diaz, C., Echevarria, L., Mendonca, C., Hernandez, F. y De Boni, L. (2013). Two-Photon Circular-Lineal Dichroism of Perylene in Solution: A Theoretical-Experimental Study. The Journal of Physical Chemistry, 117, 2742-2747. 